\title{
Long-Pulsed Modulation Regimes of Subterahertz Nanosecond Waveguide Switches
}

\author{
Maxim L. Kulygin $^{1}$, G. G. Denisov ${ }^{1,2}$, A. P. Fokin ${ }^{1}$, E. A. Novikov ${ }^{1}$, \\ S. H. Salahetdinov ${ }^{1}$, S. V. Shubin ${ }^{1}$, I. A. Litovsky ${ }^{2}$ \\ ${ }^{1}$ Institute of Applied Physics RAS, Nizhny Novgorod, Russia, kmaxim@appl.sci-nnov.ru \\ ${ }^{2}$ Lobachevski State University, Nizhny Novgorod, Russia
}

\section{INTRODUCTION}

Sub-terahertz resonator cavity-based switches [1, 2] with an active semiconductor element of Gallium Arsenide (GaAs) driven by laser pulses are used to cut microwave signal from a generator to series of wave packets. Intrinsic properties of Gallium Arsenide crystal help to achieve nanosecond level of switching performance with green driving laser emission [3]. The resonant waveguide cavity construction ensures low phase distortion to the packets' at the output [4]. The output phases are linked to each other once the input microwave signal is coherent, e.g. a gyrotron in a phase stabilization regime $[4,5]$. The prospective application of the switches is the new generation of DNP-NMR (Dynamic Nuclear Polarization - Nuclear Magnetic Resonance) spectroscopy featuring low power on probing objects and expanded resolution.

\section{OVERVIEW OF THE SWITCH}

A photo of the $260 \mathrm{GHz}$ waveguide semiconductor switch used for the second-long pulse modes is shown at Fig. 1. Microwave input and output are symmetrical IEA WR3 standard rectangular waveguides $-0.864 \times 0.432 \mathrm{~mm}$ - to the left and right flanges shaped according to UG 387 international standard. There is a rectangular input hole with the dimensions of about $0.55 \mathrm{~mm}$ in the center for driving laser pulses. A semiconductor plate of gallium arsenide is installed right under the laser input hole. The switch can be slightly fine-tuned by about a $10 \mathrm{GHz}$ with a mechanical tuning screw (not shown on the picture).

\section{MICROWAVE POWER MEASUREMENT PROBLEM}

Testing the switch for the first time demonstrated safe operation with no heating issues with about 30 $\mathrm{mW}$ test power source at the input. The maximum possible microwave power the switch can commutate is limited by an IEA WR3 waveguide standard safety value for $260 \mathrm{GHz}$ frequency band; it is about $20 \mathrm{~W}$, about two times lower than the breakdown value. A trivial way to raise the power up to the maximum of 20 $\mathrm{W}$ is a challenge because of very limited measurement precision. Modern gyrotrons are usually designed to provide power up to kilo- and megawatts, and commonly used power meters (or wattmeters) are calorimeters rated to the corresponding maximum power levels. They produce errors of worse than $100 \%$ for $20 \mathrm{~W}$ and lower powers. The lower powers are possible to register with semiconductor detectors.

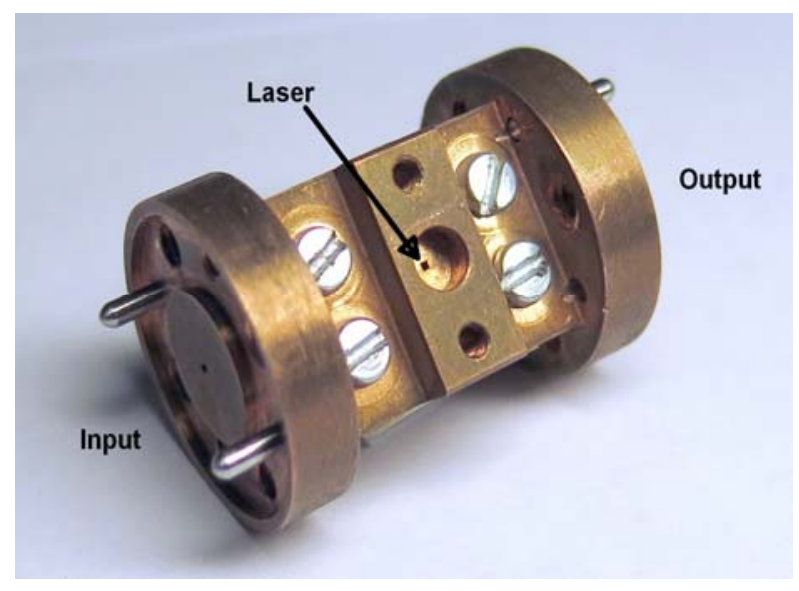

Fig. 1. Photo of the $260 \mathrm{GHz}$ resonant cavity-based switch.

But there are still problems of matching the detectors preventing spurious reflection. Up to now there are no high-precision commercial wattmeters for low powers for frequency band of $260 \mathrm{GHz}$ [6].

\section{CHANGING THE PROBLEM TO LASER MEASUREMENTS}

Meanwhile, the most of lasers are known for more than 50 years, and their power is easy to measure with high precision in wide ranges of powers for common wavelengths. So substituting the Ohmic heating mechanism by commutated microwaves with an appropriate laser absorption in the semiconductor, we can recalculate the laser power value to the corresponding microwave one. The semiconductor plate heated by an infrared laser demonstrates the same temperature distribution as it is heated by a commutated microwaves. Both the functions decay smoothly into the depth, and the maximum values for the functions are achieved at the plate's surface. For a $1.06 \mu \mathrm{m}$ laser it is known that about $R=1 / 3$ of the power is reflected from gallium arsenide, and the rest is absorbed in the semiconductor. The microwave power insertion loss coefficient for 260 GHz switches is about $-0.5 \mathrm{~dB}$, i.e. about $T=10 \%$. Let us consider the switch is able to endure the maximum laser power of $P_{L}$. So, for the maximum endurable microwave power we can write:

$$
P_{M}=\frac{(1-R)}{T} \cdot P_{L} \sim 7 \cdot P_{L}
$$

The experiments have shown that the $260 \mathrm{GHz}$ waveguide semiconductor switches designed for nanoseconds are able to make pulses up to a second. Therefore the switches cover at least nine orders by duration, 
from 1 nanosecond to 1 second and even longer. The switching capabilities at longer durations depend on the pulse length and form, the power and energy of the laser pulse, and the microwave power the switch commutates. There is no case that should be called optimum for switching; it always depends on an application the switch is used within. The maximum commutated microwave power the switch should withstand can be estimated with a substitution of two heating mechanisms in gallium arsenide, the infrared laser one and the microwave Joule one. The switch has successfully passed a 1-second 1-Watt laser pulse, and 1 second is much more than all the relaxation times in the switch. So we can estimate the switch should withstand:

$$
P_{M}=7 \cdot P_{L}=7 \mathrm{~W}
$$

The maximum safety transmittable power through an IEA standard WR3 waveguide, $0.864 \times 0.432 \mathrm{~mm}$, is about $20 \mathrm{~W}$. While we have seen the semiconductor plate has damaged after the $20 \mathrm{~W}$ laser experiment, we have managed to capture the moment of blow and conclude the minimum operable pulse duration is $15 \mathrm{~ms}$ under that maximum laser power.

The switch has been repaired after the blow and further experiments have been performed with a $1 \mathrm{kHz}$ pulsed laser. The laser has been shuttered on and off with an optical gate with operating durations from 100 $\mu \mathrm{s}$ to $1 \mathrm{~ms}$ to support different heating regimes of the semiconductor plate to make future blowouts virtually impossible.

\section{THE VERY FIRST EXPERIMENT WITH GYROTRON}

To confirm these simple theoretical estimations, (1) and (2), we performed a plain test with a gyrotron. The gyrotron [5] provides second-long microwave pulses at the frequency of about $258 \mathrm{GHz}$ with the power rate of about $100 \mathrm{~W}$ and Gaussian beam aperture of about 35 $\mathrm{mm}$. The switch has an input rectangular waveguide with dimensions $0.864 \times 0.432 \mathrm{~mm}$ corresponding to IEA WR3 standard. So to obtain the input power of several Watts we have attached a 7 x $4 \mathrm{~mm}$ horn to the input of the switch. Therefore the estimated power in the real experiment is about $3.5 \mathrm{~W}$. The maximum length of the gyrotron's pulse is $8 \mathrm{~s}$; it was limited by the power transmission line and cooling. No laser has been used so the switch is always in the ground state.

Fig.2 displays the main characteristic of the switch in the ground state when the switch is closed and the microwave power does not pass through, the insertion loss before and after the gyrotron's microwave pulse. We can see that after the pulse the main resonance became deeper, about $-5.5 \mathrm{~dB}$ compared to about $-4.7 \mathrm{~dB}$ before the test. The resonance always vanishes if the semiconductor plate is burned out. But now we can see quite a contrary case. It can be explained the way the semiconductor plate has not been burnt out under the $3.5 \mathrm{~W} 8 \mathrm{~s}$ gyrotron pulse, it even got better since only the remained dirt (occasional fingertips, organics and dust from the air) has been burnt out.

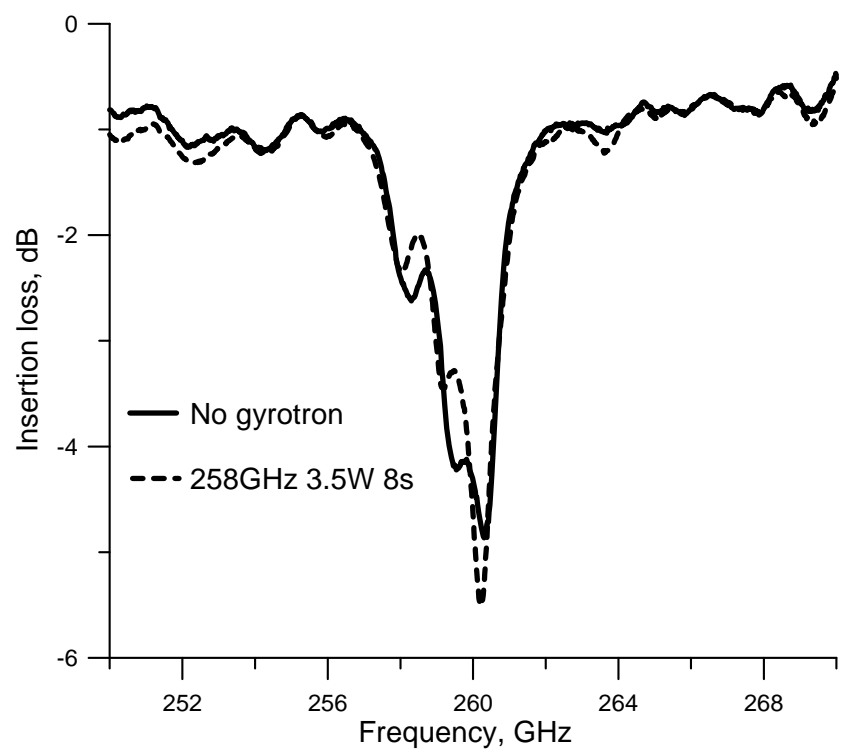

Fig. 2. Insertion loss of the switch in the ground state, solid line is before the gyrotron pulse, dashed line is after the pulse.

The switch has successfully passed the test and we can conclude that the gyrotron's radiation can be useful for final cleaning of the switch.

\section{CONCLUSION}

The second-long pulses have been produced with the switches intended for nanosecond-long pulses. So the switches have demonstrated the pulse range of about 10 orders, from $800 \mathrm{ps}$ to $8 \mathrm{~s}$ at $260 \mathrm{GHz}$. Using the switches at the second-long pulse regimes is more important than just a scientific curiosity. It provides the ability to overcome the shortage of low-precision power measurement in sub-terahertz region by changing the problem to well-known low-cost commercial laser measurements that are easily available in every laser-equipped laboratory. We have predicted theoretically our switch is tough to withstand no less than $7 \mathrm{~W}$ of microwave commutated power. And we have proven it partially in the experiment with $3.5 \mathrm{~W}$ gyrotron. The switch has not been damaged; it's even got better quality. So the gyrotron test should be included in the switch production sequence further to improve quality.

The project is supported by the Russian Foundation for Basic Research, Grant No. 15-08-03158.

\section{References}

1. Kulygin M., Denisov G. Nanosecond Laser-Driven Semiconductor Switch for $70 \mathrm{GHz}$ Microwave Radiation // J. Infrared mm THz w., V. 33, No. 6, p. 638-648.

2. Kulygin M. L., et al. Development of Waveguide Semicond. Switch. of Microw. Rad. in the 70- and 260-GHz Ranges // Radiophys. Quant. El., V. 57, No. 7, p. 509-518.

3. Kulygin M., Denisov G., Vlasova K., et al. Nanosecond Microwave Semiconductor Switches for 258...266 GHz // J. Infrared mm THz w., V. 36, No. 9, p. 845-855.

4. Kulygin M. L., Shubin S. V., et al. $260 \mathrm{GHz}$ LaserDriven Semiconductor Switches with Performance Rate up to Nanosecond. Proc. IEEE COMCAS 2015 Int. Conf.

5. Glyavin M., et al, Gyrotron Freq. Stab. by a Weak Ref. W. // Radiophys. Quant. El., V. 59, No. 9, p. 673-683.

6. Kulygin M., et al. Subterahertz Nanosecond Switches Driven by Second-Long Laser Pulses // IEEE Trans. THz Sci. Tech., V. 7, No. 2, p. 225-227. 